\title{
Prognostic value of D-lactate dehydrogenase in patients with clear cell renal cell carcinoma
}

\author{
YUE WANG ${ }^{1,2^{*}}$, GAOXIANG LI ${ }^{1,2^{*}}$, FANGNING WAN $^{1,2}$, BO DAI $^{1,2}$ and DINGWEI YE ${ }^{1,2}$ \\ ${ }^{1}$ Department of Urology, Fudan University Shanghai Cancer Centre; ${ }^{2}$ Department of Oncology, \\ Shanghai Medical College, Fudan University, Shanghai 200032, P.R. China
}

Received September 1, 2016; Accepted September 28, 2017

DOI: $10.3892 / 01.2018 .8782$

\begin{abstract}
Lactate dehydrogenase (LDH) is an enzyme involved in anaerobic glycolysis and is associated with the prognosis of patients with renal cell carcinoma (RCC). The human genome has four LDH genes: $L D H A, L D H B, L D H C$ and $L D H D$. In order to determine which of these four LDH genes may predict clear cell RCC (ccRCC), a total of 509 patients with ccRCC from The Cancer Genome Atlas (TCGA) cohort and 192 patients with ccRCC from the Fudan University Shanghai Cancer Centre (FUSCC) cohort were enrolled in the present study. The expression profiles of $L D H D$ genes in the TCGA cohort were obtained from the TCGA RNAseq database. The Cox proportional hazards regression model and Kaplan-Meier curves were used to assess relative factors. The LDH family genes that were revealed to have an association with overall survival (OS) were further validated in the FUSCC cohort. In the TCGA cohort, following Cox proportional hazards analysis, $L D H D$ expression $(\mathrm{P}=0.0400$; hazard ratio, 0.872 ; 95\% confidence interval, 0.764-0.994) was revealed to be predictive of the prognosis of patients with ccRCC. Further analysis revealed that low $L D H D$ expression $(\mathrm{P}<0.0001)$ was significantly associated with a poor prognosis in terms of OS. Additionally, the expression of LDHD $(\mathrm{P}<0.0001)$ was significantly different in patients with ccRCC compared with paired controls. In the FUSCC cohort, low $L D H D$ expression was also associated with a poor OS $(\mathrm{P}=0.0103)$, and the tumour pathological $\mathrm{T}$ stage was a factor that influenced the expression of $L D H D(\mathrm{P}=0.0120)$. Furthermore, the expression of $L D H D$ influenced the serum $L D H$ level $(\mathrm{P}=0.0126)$. The
\end{abstract}

Correspondence to: Professor Bo Dai or Dr Dingwei Ye, Department of Urology, Fudan University Shanghai Cancer Centre, 270 Dong An Road, Shanghai 200032, P.R. China

E-mail: bodai1978@126.com

E-mail: dwyeli@163.com

*Contributed equally

Key words: clear cell renal cell carcinoma, D-lactate dehydrogenase, prognosis downregulation of $L D H D$ expression may be a predictor of poor prognosis in patients with ccRCC.

\section{Introduction}

Renal cancer is a metabolic disease that starts in the cells of the kidney (1) and is responsible for $\sim 3 \%$ of all malignancies in adults (2). Globally, 250,000 new cases of kidney cancer are diagnosed each year (3). Renal cell carcinoma (RCC) accounts for $\sim 90 \%$ of all renal cancer cases $(4,5)$, and is the most common form of adult kidney cancer, possessing unique genetic and histological features. Radical nephrectomy (RN) is effective in curing early and local RCC, however, one-third of all patients present with metastatic disease at diagnosis (6). Furthermore, $20-40 \%$ of patients with RCC who undergo surgical nephrectomy will develop metastasis. Despite previous developments in therapy, there remains no effective treatment for patients with advanced stages of the disease, and RCC is generally resistant to standard chemotherapy and radiotherapy. The 5-year survival rate of patients with metastatic RCC is $<10 \%(1,7)$. Important prognostic factors of RCC include histological subtype, nuclear grade, tumour size and evidence of metastatic disease at presentation (8). In order to further our understanding on the prognosis of RCC and in order to develop novel biological therapeutic methods, the identification of molecular markers, which are useful in assisting in the management of the patients, and novel molecular targets for adjuvant therapies, is necessary.

Lactate dehydrogenase ( $\mathrm{LDH})$ is a tetrameric enzyme, belonging to the 2-hydroxy acid oxidoreductase family (9); it is a metabolic enzyme that catalyses the interconversion of pyruvate and lactate during the processes of glycolysis and gluconeogenesis depending on nutrient availability. Previous studies have revealed that pre-treatment serum LDH is a statistically significant prognostic factor in breast, renal, lung and gastric cancer (10-13). LDH is under the translational control of the hypoxia-inducible factor and MYC proto-oncogene, BHLH transcription factor (MYC), and is thus regulated by key oncogenic processes. Previous studies have revealed that pre-treatment serum LDH is a significant prognostic factor in high-risk patients with metastatic RCC (14,15). In addition, the activation of these oncogenic pathways resulted in high serum LDH levels, which were associated with drug resistance (14). 
The human genome contains four LDH genes: $L D H A$, $L D H B, L D H C$ and $L D H D(16)$. The LDH family may increase the rate of the simultaneous interconversion of pyruvate to lactate and nicotinamide adenine dinucleotide (NAD)H to NAD+ by 14 orders of magnitude $(17,18)$. Numerous genes and proteins associated with apoptosis or tumour survival have been reported to be associated with LDH activity $(19,20)$. However, the majority of previous studies on renal cancer focused on serum LDH, and few studies have analysed specifically which LDH gene serves a key function in RCC.

\section{Materials and methods}

LDH expression data. LDH expression and clinical data from The Cancer Genome Atlas (TCGA) database were sourced from the Cancer Genomics Browser of University of California Santa Cruz (https://genome-cancer.ucsc.edu/). A total of 6 members of the LDH family are included in the database, including $L D H A, L D H B, L D H C, L D H D$, LDH A like 6A (LDHAL6A) and $L D H A L 6 B$. In total, 509 primary clear cell RCC (ccRCC) tumours from patients with detailed LDH expression data obtained between January 1998 and December 2013 were selected from the updated TCGA database according to parameters defined in a previous study (21). Patients with fully characterized tumours, intact overall survival (OS) data, complete RNAseq information and those without pre-treatment were included. Data on clinicopathological characteristics, including age, sex, tumour size, Tumour-Node-Metastasis (TNM) stage (22), Fuhrman grade (23), AJCC renal cancer stage (24), laterality, haemoglobin level, white blood cell count, platelet level and OS time were collected. A follow-up of the patients was completed, with a median length of 1,063 days. During the follow-up, 347 patients succumbed.

Patient enrollment. From the Fudan University Shanghai Cancer Centre (FUSCC) cohort, a total of 192 patients with ccRCC who underwent RN or nephron-sparing nephrectomy between 2007 and 2011 were retrospectively enrolled. All the tissue samples were collected during surgery and stored at $-70^{\circ} \mathrm{C}$ in the tissue bank of the FUSCC. The pathological subtypes were determined by two pathologists, who were genitourinary specialists. Data on the clinicopathological characteristics, including sex and tumour size, were collected.

Reverse transcription-quantitative polymerase chain reaction $(R T-q P C R)$. RNA extraction, RT and RT-qPCR analysis were performed on the FUSCC cohort. In the FUSCC cohort, total RNA was isolated from the 192 ccRCC samples using TRIzol $^{\circledR}$ reagent (cat no. 15596-026; Invitrogen, Thermo Fisher Scientific, Inc., Waltham, MA, USA). A PrimeScript RT reagent kit (cat no. K1622; Thermo Fisher Scientific, Inc.) was used according to the manufacturer's protocol to synthesize first-strand cDNA from $1 \mu \mathrm{g}$ total RNA isolated from renal carcinoma cells. Serial dilutions of cDNA were amplified by qPCR using gene-specific primers. The most concentrated sample contained cDNA derived from $1 \mathrm{ng}$ total RNA. Next, SYBR Green Real-Time PCR assays (Thermo Fisher Scientific, Inc.) were performed using an ABI 7900HT (Applied Biosystems, Thermo Fisher Scientific, Inc.). The expression level of RNA was normalized to the level of $\beta$-actin. The primers for RT-qPCR analysis were synthesized by Sangon Biotech Co., Ltd. (Shanghai, China), and the sequences used were as follows: $L D H D$ forward, 5'-CAAAGCCAGGGA GGGGAAGAG-3' and reverse, 5'-CGTAGTCAGGGAACT TGTGGG-3': LDHAL6B forward, 5'-TTCCGAGAAGCC CGTTCATC-3' and reverse, 5'-GTGAAAGGGCTGCCATGT TG-3': And $\beta$-actin forward, 5'-AGCGAGCATCCCCCAAAG TT-3' and reverse, 5'-GGGCACGAAGGCTCATCATT-3'. The efficiency of amplification of the target gene ( $L D H$ family members) and the internal control ( $\beta$-actin) was examined using qPCR and TaqMan detection. Thermocycling conditions were as follows: i) Initial denaturation at $94^{\circ} \mathrm{C}$ for $4 \mathrm{~min}$; ii) 30 cycles of denaturation at $94^{\circ} \mathrm{C}$ for $30 \mathrm{sec}$, annealing at $55^{\circ} \mathrm{C}$ for $25 \mathrm{sec}$ and extension at $72^{\circ} \mathrm{C}$ for $45 \mathrm{sec}$; and iii) a final extension at $72^{\circ} \mathrm{C}$ for $5 \mathrm{~min}$ (25). Quantification was performed using the $2^{-\Delta \Delta \mathrm{Cq}}$ method (25). TNM, tumor grade, tumor stage, serum LDH level and tumor position were obtained from electronic records. Patients received regular follow-ups via telephone call or in the clinic once every 3 months. Events, including tumor recurrence, progression, metastasis and mortality were recorded.

Statistical analysis. The disease-free survival time was calculated from the date of diagnosis until the date of mortality from any cause or first recurrence. The OS time was defined as the time of diagnosis until the date of the last follow-up or mortality from any cause. Patients without recurrence events or mortality were recorded as censored at the time of the last follow-up. Statistical analysis was performed using Stata software (version 12.0; StataCorp LP, College Station, TX, USA). All statistical tests were two-tailed, and $\mathrm{P}<0.05$ was considered to indicate a statistically significant difference. Survival curves were constructed using Kaplan-Meier curves and a log-rank test in order to assess the differences between the groups. Adjusted odds ratios with $95 \%$ confidence intervals (CIs) were calculated using Cox proportional hazards models. Univariate and multivariate Cox proportional hazards analysis assessed the expression of LDH family members and OS for patients with ccRCC in the TCGA cohort. Genes known to be associated with OS were further studied. Multivariate logistic regression was performed in order to further study factors that may affect the expression of LDHs. Student's t-test or Wilcoxon's signed rank test were used for 70 paired patients in order to reveal potential differences in the expression of LDH family members between patients with ccRCC and a control population. Spearman's correlation analysis was used to determine the correlation between LDH levels and the expression of $L D H D$.

\section{Results}

Clinical characteristics of patients with ccRCC. In the TCGA cohort, the median age of the 509 patients with ccRCC was 61 years (range, 26-90 years). A total of 328 (64.4\%) patients were male and $181(35.6 \%)$ patients were female. The median follow-up time of this cohort was 35.4 months. TNM, tumor size, tumor grade, tumor stage, laterality, white blood cell count, hemoglobin level and platelet level are presented in Table I. 
Table I. Clinicopathological characteristics of patients in the TCGA and FUSCC cohorts.

\begin{tabular}{|c|c|c|}
\hline Variables & TCGA cohort & FUSCC cohort \\
\hline Total number of patients, $\mathrm{n}(\%)$ & 509 & 192 \\
\hline Median age (range), years & $61(26-90)$ & $55.5(17-84)$ \\
\hline \multicolumn{3}{|l|}{ Sex, n (\%) } \\
\hline Male & $328(64.44)$ & $131(68.23)$ \\
\hline Female & $181(35.56)$ & $61(31.77)$ \\
\hline \multicolumn{3}{|l|}{ Fuhrman grade, n (\%) } \\
\hline 1 and 2 & $234(45.97)$ & $79(41.15)$ \\
\hline 3 and 4 & $271(53.24)$ & $113(58.85)$ \\
\hline Gx & $4(0.79)$ & $0(0.00)$ \\
\hline Mean longest dimension (range), $\mathrm{cm}$ & $5.30(0-20.0)$ & $5.01(1-16.0)$ \\
\hline \multicolumn{3}{|l|}{$\mathrm{T}, \mathrm{n}(\%)$} \\
\hline $\mathrm{T} 1$ & $258(50.69)$ & $129(67.19)$ \\
\hline $\mathrm{T} 2$ & $63(12.38)$ & $29(15.10)$ \\
\hline $\mathrm{T} 3$ & $178(34.97)$ & $27(14.06)$ \\
\hline $\mathrm{T} 4$ & $10(1.96)$ & $7(3.64)$ \\
\hline \multicolumn{3}{|l|}{$\mathrm{N}, \mathrm{n}(\%)$} \\
\hline N0 & $228(44.79)$ & $181(94.27)$ \\
\hline N1 & $18(3.54)$ & $4(2.08)$ \\
\hline $\mathrm{Nx}$ & $263(51.67)$ & $7(3.64)$ \\
\hline \multicolumn{3}{|l|}{$\mathrm{M}, \mathrm{n}(\%)$} \\
\hline M0 & $406(79.76)$ & $184(95.80)$ \\
\hline M1 & $78(15.32)$ & $7(3.60)$ \\
\hline Mx & $25(4.91)$ & $1(0.50)$ \\
\hline \multicolumn{3}{|l|}{ Stage, n (\%) } \\
\hline I & $253(49.71)$ & $130(67.71)$ \\
\hline II & $51(10.02)$ & $30(15.62)$ \\
\hline III & $125(24.56)$ & $23(11.98)$ \\
\hline IV & $80(15.72)$ & $9(4.69)$ \\
\hline \multicolumn{3}{|l|}{ Laterality, n (\%) } \\
\hline Left & $239(46.95)$ & $90(46.87)$ \\
\hline Right & $269(52.85)$ & $94(48.95)$ \\
\hline Bilateral & $1(0.20)$ & $8(41.67)$ \\
\hline Median hemoglobin (range), g/l & & $139(76-398)$ \\
\hline Elevated, n (\%) & $5(0.98)$ & $20(10.42)$ \\
\hline Normal, n (\%) & $175(34.38)$ & $86(44.79)$ \\
\hline Low, n (\%) & $251(49.31)$ & $16(8.33)$ \\
\hline Undefined, n (\%) & $78(15.32)$ & $70(36.46)$ \\
\hline Median white blood cell count (range), $10^{9} / 1$ & & $6.2(3.1-18.4)$ \\
\hline Elevated, n (\%) & $160(31.43)$ & $3(1.56)$ \\
\hline Normal, n (\%) & $251(49.31)$ & $100(52.08)$ \\
\hline Low, n $(\%)$ & $8(1.57)$ & $17(8.85)$ \\
\hline Undefined, n (\%) & $89(17.49)$ & $72(37.5)$ \\
\hline Median platelet level (range), $10^{9} / 1$ & & $219(97-450)$ \\
\hline Elevated, n $(\%)$ & $35(6.88)$ & $4(2.08)$ \\
\hline Normal, n (\%) & $342(67.19)$ & $103(53.65)$ \\
\hline Low, n $(\%)$ & $45(8.84)$ & $2(1.04)$ \\
\hline Undefined, n (\%) & $86(16.90)$ & $83(43.23)$ \\
\hline Median follow-up time (range), days & $1,063(2-3,668)$ & $1,412(117-2,245)$ \\
\hline Median serum LDH level (range), U/1 ${ }^{\mathrm{a}}$ & & $155(67-761)$ \\
\hline
\end{tabular}

${ }^{a}$ No data present in the TCGA database. TCGA, The Cancer Genome Atlas; FUSCC, Fudan University Shanghai Cancer Centre; Gx, grade cannot be assessed; T, tumour; N, node; M, metastasis; LDH, lactate dehydrogenase. 

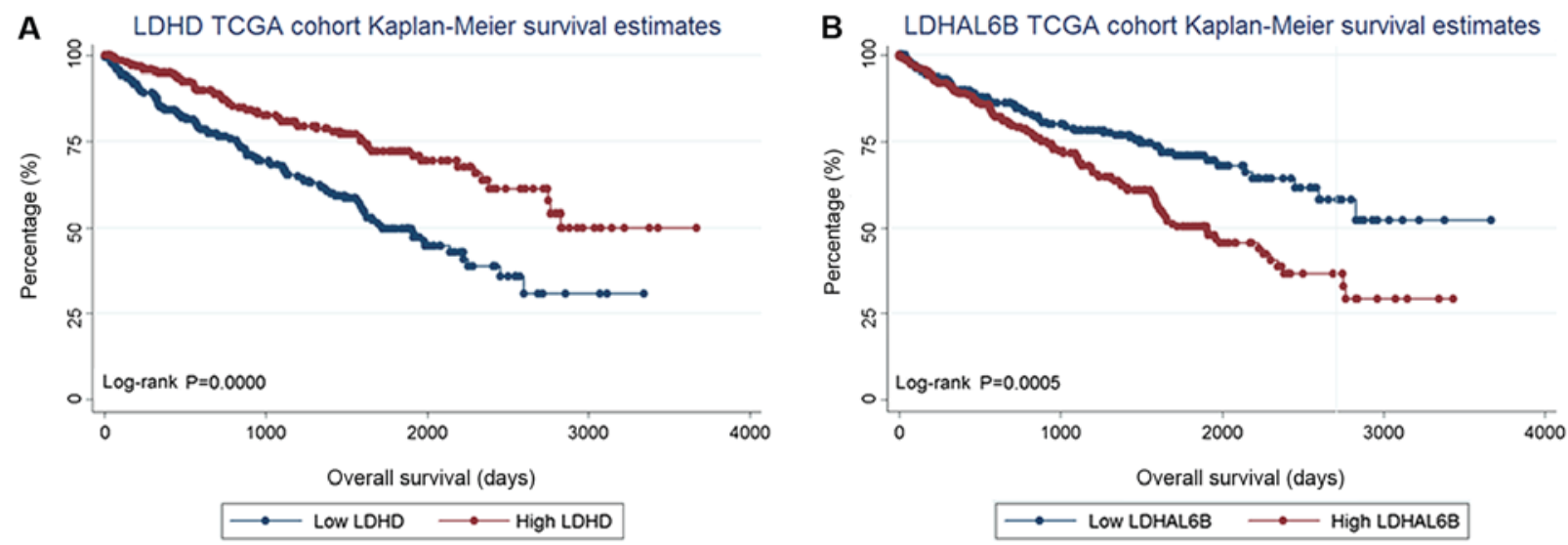

Figure 1. Kaplan-Meier plots of survival in the TCGA cohort are presented according to $L D H D$ and $L D H A L 6 B$ expression. (A) Kaplan-Meier estimates of OS are presented according to the expression of $L D H D$. (B) Kaplan-Meier estimates of OS are presented according to the expression of $L D H A L 6 B$. TCGA, The Cancer Genome Atlas; LDH, lactate dehydrogenase; LDHAL6B, LDH A like 6B; OS, overall survival.

In the FUSCC cohort, the median age of the 192 patients with ccRCC was 55.5 years (range, 17-84 years). A total of 47 patients succumbed during the follow-up, and the median follow-up time of this cohort was 47.1 months. A total of $131(68.2 \%)$ of patients were male and $61(31.8 \%)$ of patients were female. TNM, tumor size, tumor grade, tumor stage and laterality are additionally presented in Table I.

LDHD and LDHAL6B are independent prognostic factors for $O S$. In the TCGA cohort, a univariate Cox proportional hazards model was performed to assess the factors predicting OS. Age, TNM stage, Fuhrman grade, haemoglobin level, white blood cell (WBC) count, platelet (PLT) count, and $L D H C, L D H D$ and $L D H A L 6 B$ expression were significantly associated with the OS of the patients with ccRCC (all $\mathrm{P}<0.05$; Table II). A multivariate Cox analysis performed following adjustment for all the potential prognostic factors, which included age, tumor stage, Fuhrman grade, laterality, WBC count, PLT count, hemoglobin content, $L D H D, L D H C$ and LDHAL6B expression indicated that age (HR, 1.036; $95 \%$ CI, 1.020-1.053; $\mathrm{P}<0.0001)$, tumor stage (HR, 1.603; 95\% CI, 1.317-1.949; $\mathrm{P}<0.0001$ ), laterality (HR, 0.664; 95\% CI, 0.467-0.944; $\mathrm{P}=0.023)$, LDHD expression ( $\mathrm{HR}, 0.872 ; 95 \% \mathrm{CI}$, 0.764-0.994; $\mathrm{P}=0.040)$ and $L D H A L 6 B$ expression (HR, 1.285; 95\% CI, 1.048-1.576; $\mathrm{P}=0.016)$ were the only independent predictors of $\mathrm{OS}$ (all $\mathrm{P}<0.05$, Table II).

Expression quantities of LDHD and LDHAL6B are associated with prognosis and $O S$. As $\angle D H D$ and $L D H A L 6 B$ expression were the only independent predictors of OS in multivariate Cox analysis, they were selected for analysis. $L D H D$ expression and $L D H A L 6 B$ expression were revealed to be normally distributed, and were thus considered as categorical variables according to the median expression level (divided into low and high expression groups according to the median values of $L D H D$ and $L D H A L 6 B$ of 7.24 and 1.49, respectively). As a result, it was revealed that low $L D H D$ expression $(\mathrm{P}<0.0001)$ was associated with a poor prognosis for OS, whereas a low level of $L D H A L 6 B$ expression $(\mathrm{P}=0.0005)$ was associated with an improved prognosis for OS, compared with their low expression counterparts (Fig. 1). LDHD and LDHAL6B expression were considered to be categorical variables according to the median expression level. A log-rank test was performed in order to compare the survival curves between the different serum LDH levels.

In order to further understand the factors that may affect the expression of $L D H D$ and $L D H A L 6 B$, a multivariate logistic regression analysis was performed. Tumor pathological T stage was revealed to be significantly associated with $L D H D$ $(\mathrm{P}=0.003)$, whereas haemoglobin $(\mathrm{P}=0.003)$ was significantly associated with LDHAL6B expression (Table III).

The expression of LDH members in 70 paired patients from the TCGA database was then analysed to understand the difference in the expression of LDH family members between patients with ccRCC and a control group. A paired Student's t-test was used if the deviations of LDH expression between couples fitted a normal distribution, and the Wilcoxon signed rank test was employed for those that did not fit a normal distribution. In the paired Student's t-tests, the expression of LDHD $(\mathrm{P}<0.0001)$ and LDHAL6A $(\mathrm{P}<0.0001)$ were significantly different between the patients with ccRCC and the paired controls, whereas the expression of $L D H A L 6 B$ $(\mathrm{P}=0.375)$ was not significantly different. In the Wilcoxon's signed rank test analysis, the expression of $L D H A(\mathrm{P}<0.0001)$, LDHB $(\mathrm{P}<0.0001)$ and $L D H C(\mathrm{P}<0.0001)$ was significantly different between patients with ccRCC and the paired controls (Table IV).

LDHD expression is a prognostic factor for OS in the FUSCC cohort. In the FUSCC cohort, $L D H D$ and $L D H A L 6 B$ expression was validated. The expression of $\angle D H D$ and $L D H A L 6 B$ was considered as categorical variables according to the median expression level (low and high expression groups). As the expression level of genes was based on the relative values of the PCR results, patients were grouped by $\Delta-\mathrm{Cq}$ (cycle threshold). $\Delta-\mathrm{Cq}=\mathrm{Cq}$ (target genes) $-\mathrm{Cq}$ (reference genes). The median $\triangle-C q$ values of $L D H D$ and $L D H A L 6 B$ were 5.93 and 1.77 , respectively. As a result, low $L D H D$ expression was associated with a poor prognosis for $\mathrm{OS}$ (log-rank test, $\mathrm{P}=0.010$ ), whereas the expression of $L D H A L 6 B$ (log-rank test, $\mathrm{P}=0.412$ ) was not associated with OS. The Kaplan-Meier curves are presented in Fig. 2. 


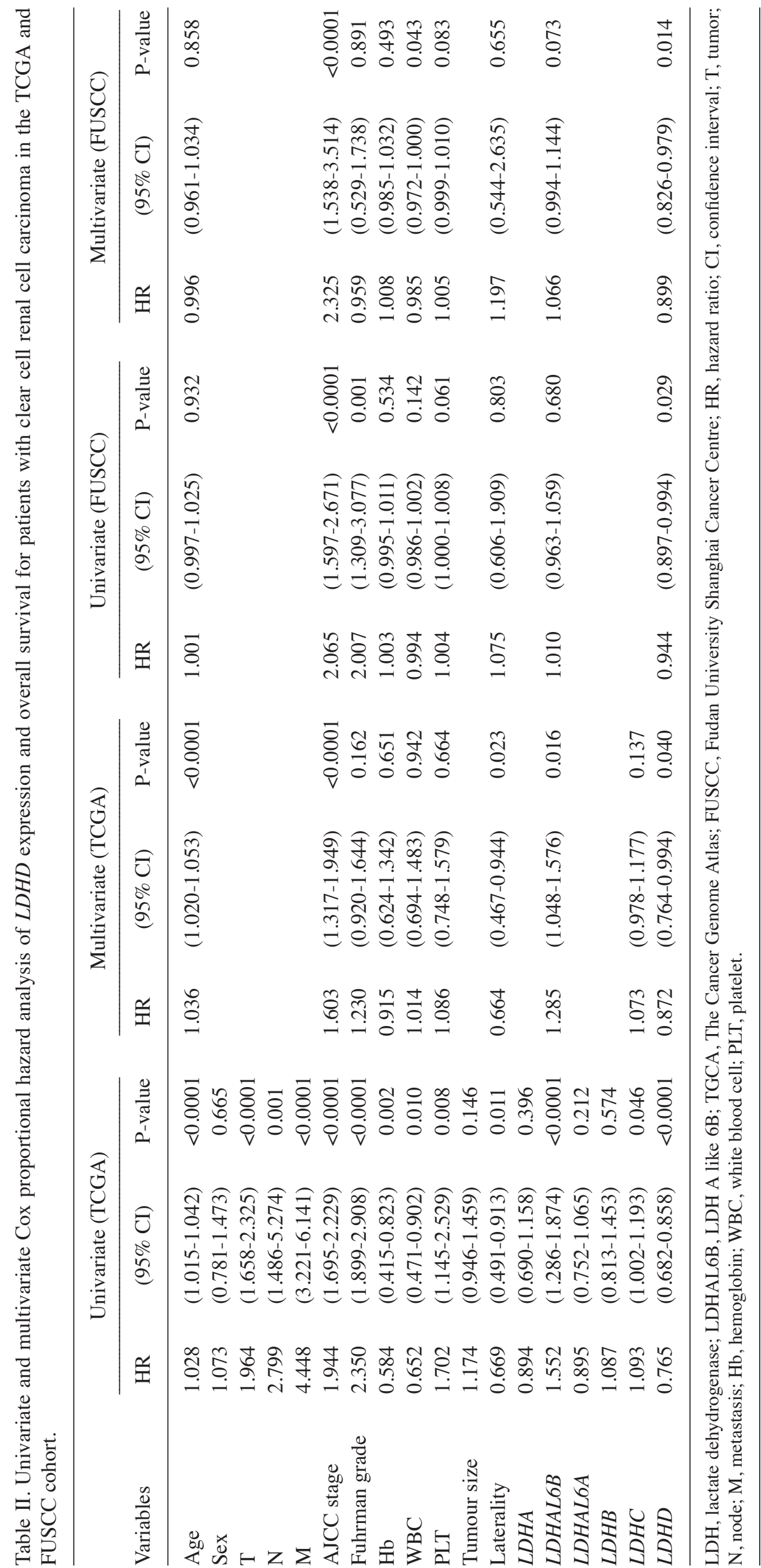


Table III. Multivariate logistic regression analysis of factors that may affect the expression of $L D H D$ and LDHAL6B in The Cancer Genome Atlas cohort with clear cell renal cell carcinoma.

\begin{tabular}{|c|c|c|c|c|c|c|}
\hline \multirow[b]{2}{*}{ Variables } & \multicolumn{3}{|c|}{$L D H D$} & \multicolumn{3}{|c|}{ LDHAL6B } \\
\hline & OR & $(95 \% \mathrm{CI})$ & P-value & OR & $(95 \% \mathrm{CI})$ & P-value \\
\hline Age & 1.013 & $(0.987-1.040)$ & 0.314 & 1.0114 & $(0.986-1.038)$ & 0.382 \\
\hline $\mathrm{T}$ & 0.565 & $(0.389-0.819)$ & 0.003 & 1.0560 & $(0.734-1.520)$ & 0.771 \\
\hline $\mathrm{N}$ & 0.965 & $(0.255-3.650)$ & 0.958 & 0.7600 & $(0.216-2.671)$ & 0.669 \\
\hline M & 1.652 & $(0.695-3.926)$ & 0.256 & 0.6730 & $(0.286-1.581)$ & 0.363 \\
\hline Fuhrman grade & 0.788 & $(0.499-1.243)$ & 0.305 & 1.0480 & $(0.665-1.649)$ & 0.841 \\
\hline $\mathrm{Hb}$ & 1.320 & $(0.700-2.488)$ & 0.391 & 0.3800 & $(0.202-0.717)$ & 0.003 \\
\hline WBC & 1.048 & $(0.564-1.948)$ & 0.881 & 0.6880 & $(0.371-1.276)$ & 0.236 \\
\hline PLT & 1.065 & $(0.564-2.011)$ & 0.847 & 1.4330 & $(0.756-2.718)$ & 0.271 \\
\hline
\end{tabular}

LDH, lactate dehydrogenase; LDHAL6B, LDH A like 6B; OR, odds ratio; CI, confidence interval; T, tumor; N, node; M, metastasis; Hb, hemoglobin; WBC, white blood cell; PLT, platelet.

Table IV. Expression of LDH family members in 70 paired patients in The Cancer Genome Atlas cohort.

\begin{tabular}{lrlr}
\hline Variable & P-value & Statistical method \\
\hline LDHA & $<0.0001$ & Wilcoxon's rank rum test & $(95 \% \mathrm{CI})$ \\
LDHAL6B & 0.3750 & Paired Student's t-test & $(0.689-1.227)$ \\
LDHAL6A & $<0.0001$ & Paired Student's t-test & $(2.269-3.168)$ \\
$L D H B$ & $<0.0001$ & Wilcoxon's rank sum test \\
$L D H C$ & $<0.0001$ & Wilcoxon's rank sum test \\
$L D H D$ & $<0.0001$ & Paired Student's t-test
\end{tabular}

LDH, lactate dehydrogenase; LDHAL6B, LDH A like 6B; CI, confidence interval.
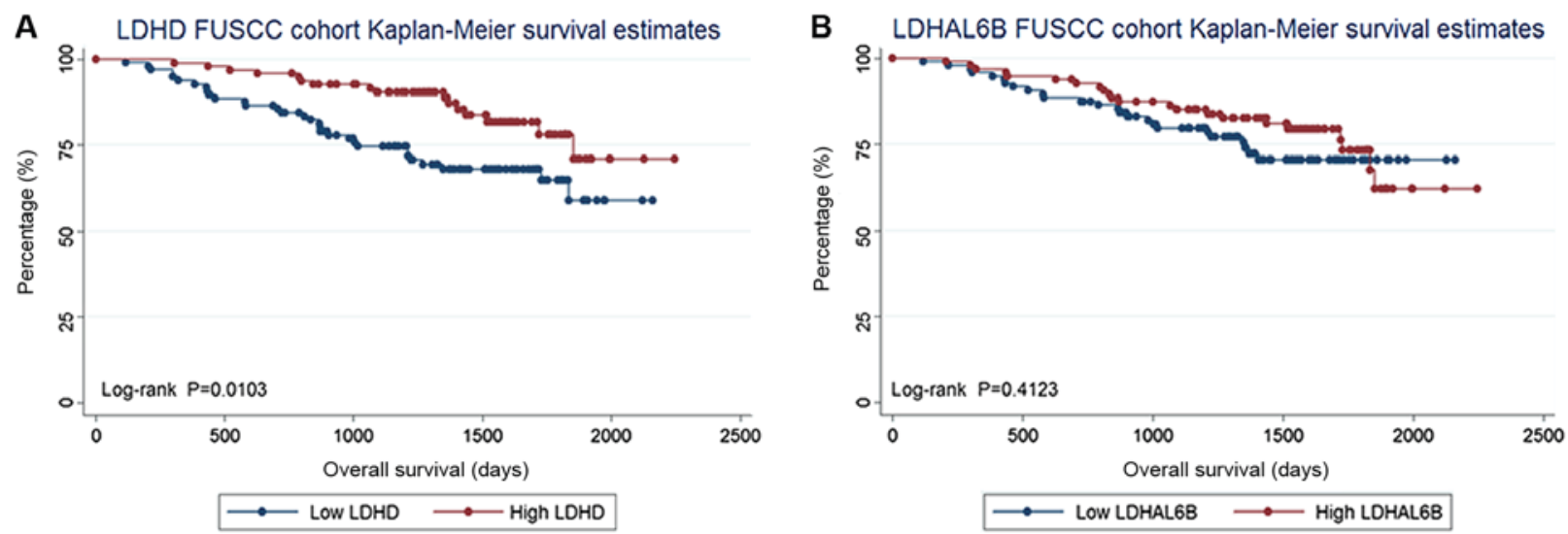

Figure 2. Kaplan-Meier plots of survival in the FUSCC cohort are presented according to $L D H D$ and $L D H A L 6 B$. (A) Kaplan-Meier estimates of OS are presented according to the expression of $L D H D$. (B) Kaplan-Meier estimates of OS are presented according to the expression of $L D H A L 6 B$. FUSCC, Fudan University Shanghai Cancer Centre; LDH, lactate dehydrogenase; LDHAL6B, LDH A like 6B; OS, overall survival.

In order to further understand the factors that may affect the expression of $L D H D$ and $L D H A L 6 B$ in the FUSCC cohort, a multivariate logistic regression analysis using the same parameters was performed. It was revealed that in the FUSCC cohort, tumor pathological $\mathrm{T}$ stage was significantly associated with the expression of $L D H D(\mathrm{P}=0.012)$, whereas age was significantly associated with the expression of $L D H A L 6 B$ $(\mathrm{P}=0.043$; Table V).

Expression level of $L D H D$ influences the serum $L D H$ level. As there was no data concerning the serum LDH level in the TCGA database, the present study tested the serum LDH of patients 
Table V. Multivariate logistic regression analysis of factors that may affect the expression of LDHD and LDHAL6B in the FUSCC cohort with ccRCC.

\begin{tabular}{|c|c|c|c|c|c|c|}
\hline \multirow[b]{2}{*}{ Variables } & \multicolumn{3}{|c|}{$L D H D$} & \multicolumn{3}{|c|}{$L D H A L 6 B$} \\
\hline & OR & $(95 \% \mathrm{CI})$ & P-value & OR & $(95 \% \mathrm{CI})$ & P-value \\
\hline Age & 0.999 & $(0.962-1.038)$ & 0.971 & 0.962 & $(0.926-0.999)$ & 0.043 \\
\hline $\mathrm{T}$ & 0.519 & $(0.312-0.864)$ & 0.012 & 0.959 & $(0.604-1.523)$ & 0.859 \\
\hline $\mathrm{N}$ & 11.272 & $(0.856-148.417)$ & 0.065 & 2.651 & $(0.223-31.479)$ & 0.440 \\
\hline $\mathrm{M}$ & 1.056 & $(0.069-16.042)$ & 0.969 & 0.521 & $(0.6038-7.080)$ & 0.624 \\
\hline Fuhrman grade & 0.587 & $(0.295-1.169)$ & 0.130 & 0.579 & $(0.300-1.117)$ & 0.103 \\
\hline $\mathrm{Hb}$ & 0.986 & $(0.958-1.013)$ & 0.305 & 1.000 & $(0.974-1.026)$ & 0.978 \\
\hline WBC & 0.997 & $(0.988-1.007)$ & 0.578 & 0.995 & $(0.984-1.006)$ & 0.373 \\
\hline PLT & 1.003 & $(0.997-1.009)$ & 0.300 & 1.001 & $(0.995-1.007)$ & 0.733 \\
\hline
\end{tabular}

LDH, lactate dehydrogenase; LDHAL6B, LDH A like 6B; FUSCC, Fudan University Shanghai Cancer Centre; OR, odds ratio; CI, confidence interval; T, tumor; N, node; M, metastasis; Hb, hemoglobin; WBC, white blood cell; PLT, platelet.

from the FUSCC cohort when they were diagnosed with a kidney tumor in order to understand whether the expression of $L D H D$ is associated with serum LDH. In this further analysis of the FUSCC cohort, the 192 patients were divided into two groups according to their serum $\mathrm{LDH}$ level. Log-rank testing revealed that a serum LDH level higher than the upper limit of normal (215 U/l) was associated with a poor prognosis for OS (log-rank test, $\mathrm{P}=0.006$; Fig. 3). This result is concurrent with those of previous studies $(11,14,21,26)$. Furthermore, Spearman's correlation analysis (data not shown) revealed that the serum LDH level has a negative correlation with the expression of $L D H D(\mathrm{P}=0.028)$. Despite the fact that the analysis of serum LDH is a protein test and the analysis of $L D H D$ is a gene test, it may be concluded that the expression of $L D H D$ is associated with the levels of serum LDH.

Expression of LDHD and LDHAL6B is not associated with recurrence-free survival (RFS) and cancer-specific survival (CSS) in the FUSCC cohort. In order to understand if the expression of $L D H D$ and $L D H A L 6 B$ were associated with RFS and CSS, $L D H D$ and $L D H A L 6 B$ expression in the FUSCC cohort was considered as a categorical variable according to the median expression level, and divided into high and low expression groups as aforementioned. RFS was measured from the date of renal resection until the date of radiographic detection of recurrence or the last follow-up. CSS was measured from the date of renal resection until the the date of mortality due to renal cancer or the last follow-up. A total of 18 patients were diagnosed with radiographic recurrence and 10 patients succumbed to renal cancer during the follow-up. As a result, it was revealed that the expression of $L D H D$ was not associated with RFS (log-rank test, $\mathrm{P}=0.887$ ) or CSS (log-rank test, $\mathrm{P}=0.133)$. LDHAL6B expression was also not associated with RFS (log-rank test, $\mathrm{P}=0.364)$ and CSS (log rank test, $\mathrm{P}=0.430$ ) (data not shown).

\section{Discussion}

In the present study, it was demonstrated that certain LDH gene family members were associated with the OS of patients

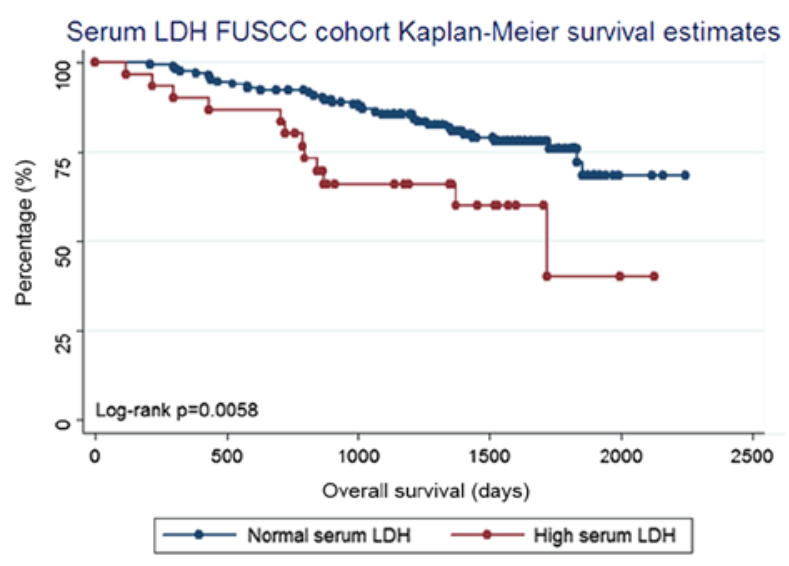

Figure 3. Kaplan-Meier plots of overall survival in the FUSCC cohort are presented according to serum LDH. FUSCC, Fudan University Shanghai Cancer Centre; LDH, lactate dehydrogenase.

with ccRCC. Members of this family, particularly $L D H D$ and $L D H A L 6 B$, were independent prognostic factors for the OS of patients with ccRCC in the present study.

The human genome has four LDH genes: $L D H A, L D H B$, $L D H C$, and $L D H D$. Of these genes (26), $L D H B$ and $L D H C$ are $\mathrm{L}$ isomers and $L D H D$ is a $\mathrm{D}$ isomer (27).

LDHD metabolism has been demonstrated to occur in mitochondria, but its function in cancer is unclear. Previous studies have demonstrated that in prostate cancer cells, the metabolism of D-lactate inside mitochondria via $L D H D$ and the metabolic activity of $L D H D$ in tumor cells were higher compared with that in normal cells $(28,29)$. Furthermore, the LDHD protein level and activity are higher in cancerous cells compared with that in normal prostate cells (30). LDHD is a unique gene; in Sporolactobacillus inulinus strain CASD, LDHD may use NADH and NADPH, but preferentially uses NADPH as a coenzyme, which is different from the coenzyme utilization of other LDHs $(31,32)$.

A feature of tumor cells is their reliance upon fermentative glycolysis, a common phenomenon coined 'the Warburg 
effect' (33). LDH isoenzymes are metabolically regulated, and are linked to glycolysis and the Warburg effect (34). Previous studies have revealed that the level of pre-treatment serum $\mathrm{LDH}$ is a significant prognostic factor in numerous types of cancer, including RCC (35-37). LDH is under the translational control of hypoxia-inducible factor and MYC, and is thus regulated by key oncogenic processes $(33,38)$. However, in the field of oncology, little is known concerning the subtypes of the LDH family. The few previous studies focused on this topic have been mainly confined to the cellular level. RCC is a disease that is closely associated with metabolism $(39,40)$. The LDH gene family serves an important function in ccRCC. $L D H A$ may possess multiple additional functions in non-neoplastic and neoplastic tissues (41). There are numerous genes and proteins, including tumor protein p53, vascular endothelial growth factor and 2'-deoxynucleoside 5'-phosphate N-hydrolase 1, amongst others, reported to be associated with $L D H A$ activity (41). The expression of $L D H A$ is associated with the prognosis of patients with brain tumors. A previous study revealed that $L D H A$ metabolic activity in brain tumor cells was stronger compared with normal cells (41). However, the clinical association between $L D H D$ expression and tumors is not clear. There are also few studies focused on renal cancer.

The present study revealed that $L D H D$ may serve an important function in the prognosis of patients with ccRCC. It was revealed that the expression of $L D H D$ and $L D H A L 6 B$ were independent prognostic factors for the OS of patients with ccRCC. The downregulated expression of $L D H D$ was associated with a poor prognosis and a shorter OS. The expression of $L D H D$ is associated with the prognosis of patients with ccRCC. In addition, the tumor stage was significantly associated with the expression of $L D H D$. In the statistical analysis of 70 paired patients, the expression of $L D H D$ was also significantly different between patients with ccRCC and paired controls. Furthermore, the serum LDH level was significantly associated with the expression of $L D H D$. The results of the present study confirmed that $L D H D$ is a useful biomarker for patients with ccRCC. In addition, the expression of $L D H D$ was associated with patient prognosis. To the best of our knowledge, no previous study has clarified the function of $L D H D$ in renal tumors. Although Girgis et al (42) had previously assessed the expression of $L D H A$ at the protein level via the use of immunohistochemistry in 385 patients and indicated that $L D H A$ upregulation may be a predictor of a poor prognosis in patients with ccRCC, in the present study, $L D H A$ expression was not significantly associated with the OS of patients with ccRCC in the TCGA cohort or the FUSCC cohort.

LDHD is a subtype of LDH and serves an important function in renal cancer. The integration of $L D H D$ expression has the potential to be a useful biomarker in the identification of poor prognosis in patients with ccRCC. Although it has potential as a novel renal cancer biomarker, its specific mechanism remains unknown. To date, LDHD, which is produced in the methylglyoxal (MG) pathway, is presumed to be released by cancer cells (43). The MG pathway produces LDHD as a final product that is largely modified in cancer cells, with a specific function in the glyoxalase systems, serving to eliminate the cytotoxic MG mainly derived from glycolysis (44). The rationally engineered LDHD may efficiently use NADH and NADPH as cofactors. Additionally, the mitochondrial metabolism of LDHD in cancer cells is more active compared with that of normal cells (45). Renal cancer is a disease that is associated with metabolism and obesity. LDHD may serve a key function in the progression and development of ccRCC.

There are a number of limitations to the present study: Firstly, the patients included were from the FUSCC, with good follow up, but patients from other centres were not included. Secondly, all the tissue specimens in the present study were sourced from patients who were suitable candidates for surgery, and the same results may not apply to patients who are not suitable candidates for surgery.

The present study has indicated at the association between ccRCC outcome and $L D H D$, however, the underlying mechanism remains poorly understood. The present study may have opened a threshold to a novel aspect of ccRCC biomarkers or therapeutic targets. Further studies are required.

In conclusion, $L D H D$ was identified as an independent prognostic factor for the OS of patients with ccRCC. Low $L D H D$ expression was associated with a poor prognosis for OS, and tumor grade was significantly associated with $L D H D$ expression. $L D H D$ may function as a tool to reveal further genes associated with prognosis in ccRCC.

\section{Acknowledgements}

The presentstudy was supported by the InternationalCooperation and Exchange of Science and Technology Commission of the Shanghai Municipality (grant no. 12410709300), the Guide Project of Science and Technology Commission of Shanghai Municipality (grant no. 124119a7300), and the Outstanding Young Talent Training Plan of Shanghai Municipal Commission of Health and Family Planning (grant no. XYQ2013102).

\section{Competing interests}

The authors declare that they have no competing interests.

\section{References}

1. Linehan WM, Srinivasan R and Schmidt LS: The genetic basis of kidney cancer: A metabolic disease. Nat Rev Urol 7: 277-285, 2010.

2. Simard EP, Ward EM, Siegel R and Jemal A: Cancers with increasing incidence trends in the United States: 1999 through 2008. CA Cancer J Clin 62: 118-128, 2012.

3. Siegel R, Naishadham D and Jemal A: Cancer statistics, 2012. CA Cancer J Clin 62: 10-29, 2012.

4. Ridge CA, Pua BB and Madoff DC: Epidemiology and staging of renal cell carcinoma. Semin Intervent Radiol 31: 3-8, 2014.

5. Chow WH, Dong LM and Devesa SS: Epidemiology and risk factors for kidney cancer. Nat Rev Urol 7: 245-257, 2010.

6. Liang L, Li L, Zeng J, Gao Y, Chen YL, Wang ZQ, Wang XY, Chang LS and He D: Inhibitory effect of silibinin on EGFR signal-induced renal cell carcinoma progression via suppression of the EGFR/MMP-9 signaling pathway. Oncol Rep 28: 999-1005, 2012.

7. Maher ER: Genomics and epigenomics of renal cell carcinoma. Semin Cancer Biol 23: 10-17, 2013.

8. Ficarra V, Galfano A, Novara G, Iafrate M, Brunelli M, Secco S, Cavalleri S, Martignoni G and Artibani W: Risk stratification and prognostication of renal cell carcinoma. World J Urol 26: 115-125, 2008 .

9. Burgner JW II and Ray WJ Jr: On the origin of the lactate dehydrogenase induced rate effect. Biochemistry 23: 3636-3648, 1984. 
10. Brown JE, Cook RJ, Lipton A and Coleman RE: Serum lactate dehydrogenase is prognostic for survival in patients with bone metastases from breast cancer: A retrospective analysis in bisphosphonate-treated patients. Clin Cancer Res 18: 6348-6355, 2012.

11. Shen J, Chen Z, Zhuang Q, Fan M, Ding T, Lu H and He X: Prognostic value of serum lactate dehydrogenase in renal cell carcinoma: A systematic review and meta-analysis. PLoS One 11: e0166482, 2016.

12. Chen Y, Zhang H, Xu A, Li N, Liu J, Liu C, Lv D, Wu S, Huang L, Yang $\mathrm{S}$, et al: Elevation of serum 1-lactate dehydrogenase B correlated with the clinical stage of lung cancer. Lung cancer 54: 95-102, 2006.

13. Wang ZX, Yang LP, Qiu MZ, Wang ZQ, Zhou YX, Wang F, Zhang DS, Wang FH, Li YH and Xu RH: Prognostic value of preoperative serum lactate dehydrogenase levels for resectable gastric cancer and prognostic nomograms. Oncotarget 7: 39945-39956, 2016.

14. Armstrong AJ, George DJ and Halabi S: Serum lactate dehydrogenase predicts for overall survival benefit in patients with metastatic renal cell carcinoma treated with inhibition of mammalian target of rapamycin. J Clin Oncol 30: 3402-3407, 2012.

15. Cetin B, Afsar B, Deger SM, Gonul II, Gumusay O, Ozet A, Benekli M, Coskun U and Buyukberber S: Association between hemoglobin, calcium, and lactate dehydrogenase variability and mortality among metastatic renal cell carcinoma. Int Urol Nephrol 46: 1081-1087, 2014.

16. Adeva-Andany M, López-Ojén M, Funcasta-Calderón R, Ameneiros-Rodríguez E, Donapetry-García C, Vila-Altesor M and Rodríguez-Seijas J: Comprehensive review on lactate metabolism in human health. Mitochondrion 17: 76-100, 2014

17. Fan J, Hitosugi T, Chung TW, Xie J, Ge Q, Gu TL, Polakiewicz RD Chen GZ, Boggon TJ, Lonial S, et al: Tyrosine phosphorylation of lactate dehydrogenase A is important for NADH/NAD(+) redox homeostasis in cancer cell. Mol Cell Biol 31: 4938-4950, 2011.

18. Markert CL, Shaklee JB and Whitt GS: Evolution of a gene. Multiple genes for LDH isozymes provide a model of the evolution of gene structure, function and regulation. Science 189: 102-114, 1975.

19. Sun Q, Chen X, Ma J, Peng H, Wang F, Zha X, Wang Y, Jing Y, Yang H, Chen R, et al: Mammalian target of rapamycin up-regulation of pyruvate kinase isoenzyme type M2 is critical for aerobic glycolysis and tumor growth. Proc Natl Acad Sci USA 108: 4129-4134, 2011.

20. Zha X, Wang F, Wang Y, He S, Jing Y, Wu X and Zhang H: Lactate dehydrogenase $\mathrm{B}$ is critical for hyperactive mTOR-mediated tumorigenesis. Cancer Res 71: 13-18, 2011.

21. Hoffmann R, Franzke A, Buer J, Sel S, Oevermann K, Duensing A, Probst M, Duensing S, Kirchner H, Ganser A and Atzpodien J: Prognostic impact of in vivo soluble cell adhesion molecules in metastatic renal cell carcinoma. Br J Cancer 79: 1742-1745, 1999.

22. Sobin LH and Fleming ID: TNM classification of malignant tumors, fifth edition (1997). Union Internationale Contre le Cancer and the American Joint Committee on Cancer. Cancer 80: 1803-1804, 1997.

23. Rioux-Leclercq N: The Fuhrman grading system for kidney cancer prognosis. Prog Urole 16 (4 Suppl FMC): S5-S8, 2006 (In French).

24. Kim SP, Alt AL, Weight CJ, Costello BA, Cheville JC, Lohse C, Allmer C and Leibovich BC: Independent validation of the 2010 American Joint Committee on Cancer TNM classification for renal cell carcinoma: Results from a large, single institution cohort. J Urol 185: 2035-2039, 2011.

25. Livak KJ and Schmittgen TD: Analysis of relative gene expression data using real-time quantitative PCR and the 2(-Delta Delta C(T)) method. Methods 25: 402-408, 2001.

26. Gallo M, Sapio L, Spina A, Naviglio D, Calogero A and Naviglio S: Lactic dehydrogenase and cancer: An overview. Front Biosci (Landmark Ed) 20: 1234-1249, 2015.

27. Gleason FH and Nolan RA: D(-)-lactate dehydrogenase in lower fungi. Science 152: 1272-1273, 1966.
28. Rojo EE, Guiard B, Neupert W and Stuart RA: Sorting of D-lactate dehydrogenase to the inner membrane of mitochondria. Analysis of topogenic signal and energetic requirements. J Biol Chem 273: 8040-8047, 1998

29. Lapierre L, Germond JE, Ott A, Delley M and Mollet B: D-Lactate dehydrogenase gene (ldhD) inactivation and resulting metabolic effects in the Lactobacillus johnsonii strains La1 and N312. Appl Environ Microbiol 65: 4002-4007, 1999.

30. de Bari L, Moro L and Passarella S: Prostate cancer cells metabolize d-lactate inside mitochondria via a D-lactate dehydrogenase which is more active and highly expressed than in normal cells. FEBS Lett 587: 467-473, 2013.

31. Pagala VR, Park J, Reed DW and Hartzell PL: Cellular localization of D-lactate dehydrogenase and NADH oxidase from Archaeoglobus fulgidus. Archaea 1: 95-104, 2002.

32. Bernard N, Johnsen K, Holbrook JJ and Delcour J: D175 discriminates between NADH and NADPH in the coenzyme binding site of Lactobacillus delbrueckii subsp. bulgaricus D-lactate dehydrogenase. Biochem Biophys Res Commun 208: 895-900, 1995.

33. Kim JW and Dang CV: Cancer's molecular sweet tooth and the Warburg effect. Cancer Res 66: 8927-8930, 2006.

34. De Saedeleer CJ, Copetti T, Porporato PE, Verrax J, Feron O and Sonveaux P: Lactate activates HIF-1 in oxidative but not in Warburg-phenotype human tumor cells. PLoS One 7: e46571, 2012.

35. von Eyben FE, Madsen EL, Liu F, Amato R and Fritsche H: Serum lactate dehydrogenase isoenzyme 1 as a prognostic predictor for metastatic testicular germ cell tumours. Br J Cancer 83: 1256-1259, 2000.

36. Halabi S, Small EJ, Kantoff PW, Kattan MW, Kaplan EB, Dawson NA, Levine EG, Blumenstein BA and Vogelzang NJ: Prognostic model for predicting survival in men with hormone-refractory metastatic prostate cancer. J Clin Oncol 21: 1232-1237, 2003

37. Agarwala SS, Keilholz U, Gilles E, Bedikian AY, Wu J, Kay R, Stein CA, Itri LM, Suciu S and Eggermont AM: LDH correlation with survival in advanced melanoma from two large, randomised trials (Oblimersen GM301 and EORTC 18951). Eur J Cancer 45: 1807-1814, 2009

38. Kim JW and Dang CV: Multifaceted roles of glycolytic enzymes. Trends Biochem Sci 30: 142-150, 2005.

39. Cho E, Giovannucci EL and Joh HK: Nutrients related to one-carbon metabolism and risk of renal cell cancer. Cancer Causes Control 24: 373-382, 2013

40. Yang OC, Maxwell PH and Pollard PJ: Renal cell carcinoma: Translational aspects of metabolism and therapeutic consequences. Kidney Int 84: 667-681, 2013.

41. Serganova I, Rizwan A, Ni X, Thakur SB, Vider J, Russell J, Blasberg R and Koutcher JA: Metabolic imaging: A link between lactate dehydrogenase A, lactate, and tumor phenotype. Clin Cancer Res 17: 6250-6261, 2011.

42. Girgis H, Masui O, White NM, Scorilas A, Rotondo F, Seivwright A, Gabril M, Filter ER, Girgis AH, Bjarnason GA, et al: Lactate dehydrogenase A is a potential prognostic marker in clear cell renal cell carcinoma. Mol Cancer 13: 101, 2014.

43. Santel T, Pflug G, Hemdan NY, Schäfer A, Hollenbach M, Buchold M, Hintersdorf A, Lindner I, Otto A, Bigl M, et al: Curcumin inhibits glyoxalase 1: A possible link to its anti-inflammatory and anti-tumor activity. PLoS One 3: e3508, 2008.

44. Rulli A, Carli L, Romani R, Baroni T, Giovannini E, Rosi G and Talesa V: Expression of glyoxalase I and II in normal and breast cancer tissues. Breast Cancer Res Treat 66: 67-72, 2001.

45. Meng H, Liu P, Sun H, Cai Z, Zhou J, Lin J and Li Y: Engineering a d-lactate dehydrogenase that can super-efficiently utilize NADPH and NADH as cofactors. Sci Rep 6: 24887, 2016.

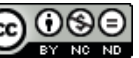

This work is licensed under a Creative Commons Attribution-NonCommercial-NoDerivatives 4.0 International (CC BY-NC-ND 4.0) License. 